
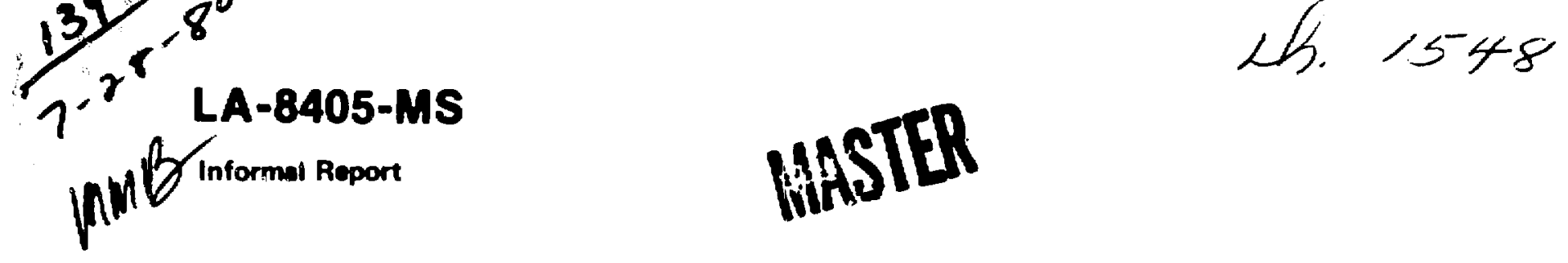

\title{
Preliminary Simulation Studies of Accelerator Cavity Loading
}

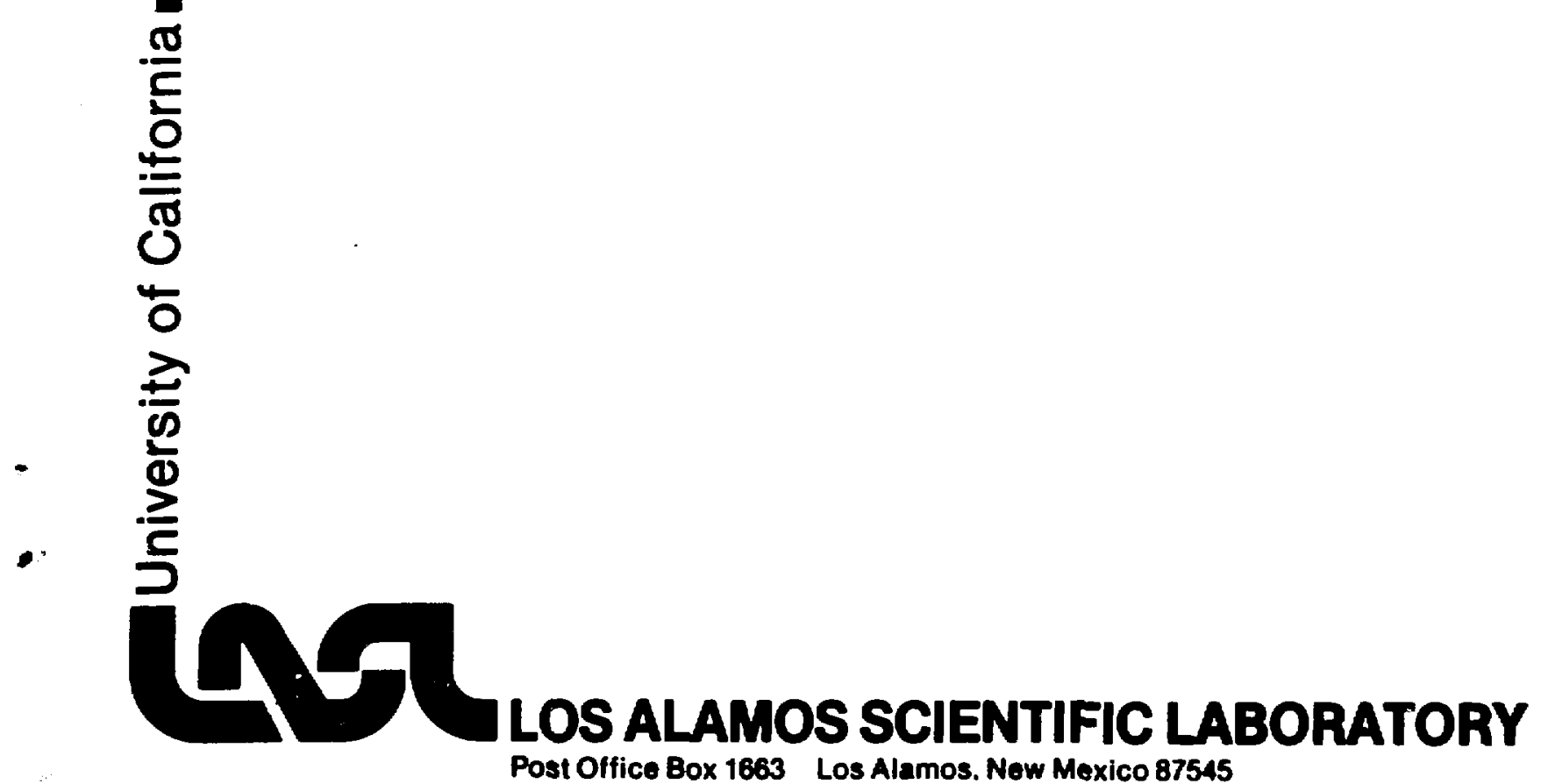




\title{
Preliminary Simulation Studies of Accelerator Cavity Loading
}

\author{
Rickey J. Faehl
}
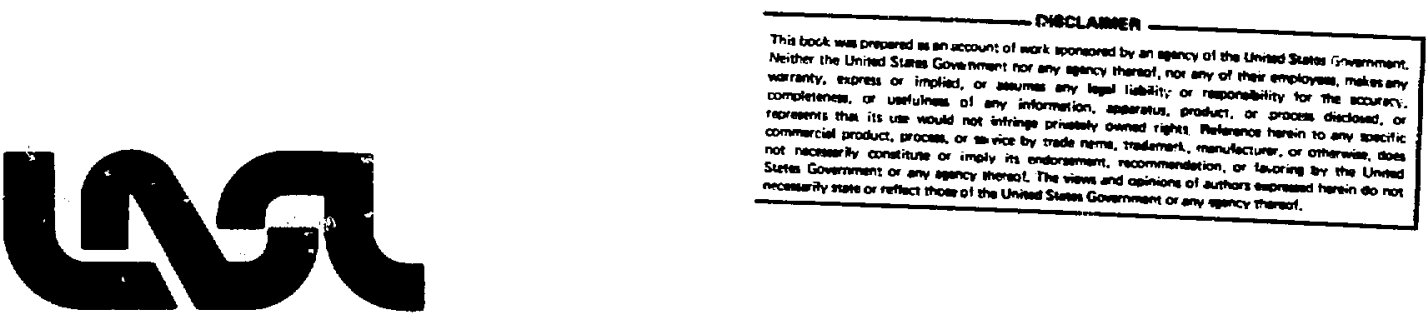


\title{
PRELIMINARY SIMULATION STUDIES OF ACCELERATOR CAVITY LOADING
}

by

Rickey J. Faehl

\begin{abstract}
Two-dimensional simulations of loading effects in a $350 \mathrm{MHz}$ accelerator cavity have been performed. Electron currents of 1-10 kA have been accelerated in $5 \mathrm{MV} / \mathrm{m}$ fields. Higher order cavity modes induced by the beam may lead to emittance growth. Operation in an autoaccelerator mode has been studied.
\end{abstract}

\section{INTRODUCTION}

Numerical studies have been undertaken to examine dynamical cavity loading in configurations similar to those found in $\mathrm{rf}-\mathrm{linacs}$ at LASL. These were preliminary and exploratory studies, representing the first attempts to our knowledge to model accelerator phys: is in two-dimensional, fully electromagnetic particle-in-cell (PIC) simulations. The CCUBE code, which has been described previously, ${ }^{l}$ solves Maxwell's equations on a fixed mesh and constructs source terms by averaging the self-consistent trajectories of a large number of "particles" onto this mesh. For these studies the primary sources were the micropulses of accelerated electrons. The cavity dimensions were appropriate to support a fundamental TM mode at $350 \mathrm{MHz}$.

\section{DESCRIPTION OF CALCULATIONS}

Relativistic electrons were chosen for the accelerating particles. With an injection energy of $2.3 \mathrm{MeV}\left(y_{0}=5.5\right)$, changes in velocity were minimal during the acceleration phase. Pulses of $0.4 \mathrm{nsec}$ duration were injected with a parabolic current form. Peak current on these pulses was $1.0 \mathrm{kA}$. To transport them, a uniform $4.0 \mathrm{kG}$ solenoidal field was imposed. This is in contrast to conventional accelerator transport schemes which rely on strong focusing. In the present approach, laminarity is maintained by injecting the pulses in states near to intense beam equilibrium. Fine tuning of the guide fields 
throughout the cavity can probably be used to reduce multi-cavity emittance growth. Even though these initial studies were not optimized, it should be noted that this system will easily accommodate $10 \mathrm{kA}$ peak currents and more.

A $350 \mathrm{MHz}$ mode was excited in the cavity of radius $32.5 \mathrm{~cm}$. Excitation was accomplished by driving a $z$-slotted antenna. (This antenna was the mathematical idealization of an array of linear antennas such that $E_{z}=0$ on the array, $E_{\theta}$ was unaffected, and the total array had no azimuthal gradients.) Cavity fields were thus rung up in a realistic fashion. This self-consistent excitation was felt to be necessary to model fringing fields in the $8 \mathrm{~cm}$ diameter drift tube on axis. Although the ring-up phase was relatively inexpensive, since no beam particles were present, economy dictated beam injection before the fieids had attained their full magnitudes. The relative change over one period, however, was less than $5 \%$. Figure 1 shows the $\mathrm{E}_{z}$ history for a probe on axis roughly in the center of the cavity. Electrons were injected only when $t \geq 0$.

The fundamental TM mode has no axial gradients. The cavity could therefore be made as long as desired. Figure 2 shows the superposition of four radial profiles of the cavity field at different axial positions. A length of $17 \mathrm{~cm}$ was chosen so that significant energy transfer occurred in one transit time. Peak fields were roughly $5 \mathrm{MV} / \mathrm{m}$ so maximum energy extraction would be $0.85 \mathrm{MeV} /$ cavity. Because the relativistic electron transit was a significant fraction of the period, $\Delta T / T=0.2$, where $T=2 \pi / \omega$, a significant variation of accelerating gradient was sampled. Of course, this would be undesirable in an actual accelerator, but our goal was to maximize loading effects.

\section{DESCRIPTION OF RESULTS}

Two calculations were conducted to determine loading effects. Both used the same ring-up calculation as an initial condition, with only the electron injection parameters different. In the first, electrons were injected to be in phase with the accelerating gradient. Figure 3 shows the energy space of a typical pulse at injection and after transit through the cavity. The energy gain is $0.56 \mathrm{MeV}$, compared to the idealized limit of $0.85 \mathrm{MeV}$. The other calculation involved pulses in phase with the deceleraing gradient, that is $180^{\circ}$ out of phase with first. In theory this latter operation could enhance the cavity fields, since energy is given from the electrons to the field. This will be discussed in more detail in the conclusions, but suffice it to say for 
now that it, in fact, behaved as expected. Figure 4 compares probe traces of the two runs at a typical off-axis position.

By taking one-half the difference between corresponding probe plots, one may gain a fairly accurate picture of the cavity loading. After transit of the first beam pulse, measurable field differences were found, out to probes of radius $R=17.5 \mathrm{~cm}$ but not at probes at $R=26 \mathrm{~cm}$. Since the cavity length is $17 \mathrm{~cm}$, it is suggestive that the value of the cavity length represents the limit of radial cavity loading effects. This is not conclusive on the basis of one configuration, however. Furthermore, after multiple pulses had been accelerated, field differences were measurable at all radii.

Another interesting feature of these calculations was the perturbation of the cavity fields by the beam(s). Figure 2 showed the fundamental mode excitation with no nulls in the absence of beam. After injection, however, higher order fields were clearly evident. Figure 5 shows a similar ensemble of profiles as in Fig. 2. Third and fourth order cavity modes have been induced. This phenomena was evident in both accelerating and decelerating calculations. Emittance growth could be associated with these unwanted modes.

IV. CONCLUSIONS

Preliminary studies of cavity loading indicate that short-term loading may be limited to a radial distance equal to the length of the cavity mouth. These effacts are distributed over the entire cavity after several field cycles, however. Emphasis should be placed on the tentative nature of the results at present. Additional calculations with minor variation in parameters would greatly reduce uncertainties here. Even with these caveats, the simulations proved to be fruitful and illuminating. Operation of the cavities in an autoaccelerator mode, for instance, seems sufficiently promising that I would strongly recommend more detailed investigations. A trial calculation with a beam c̈uration many $\mathrm{rf}$ periods long at $10 \mathrm{kA}$ showed no net field depletion. If switching techniques are feasible, it may be possible to accelerate much higher beam currents in $r f$ linacs than has previously been thought practical. Another unexpected bonus from the calculations was observation of extraneous mode excitation induced by these high-current beams. Overall, these calculations appear to have only scratched the surface of a c...ch larger body of accelerator problems to which this type of sophisticated simulation couplad with analytical calculations can make significant contributions. 


\section{REFERENCE}

1. L. E. Thode, B. B. Godfrey, and W. R. Shanahan, "Vacuum Propagation of Solid Relativistic Electron Beams," Phys. Fluids 22, 747 (1979).

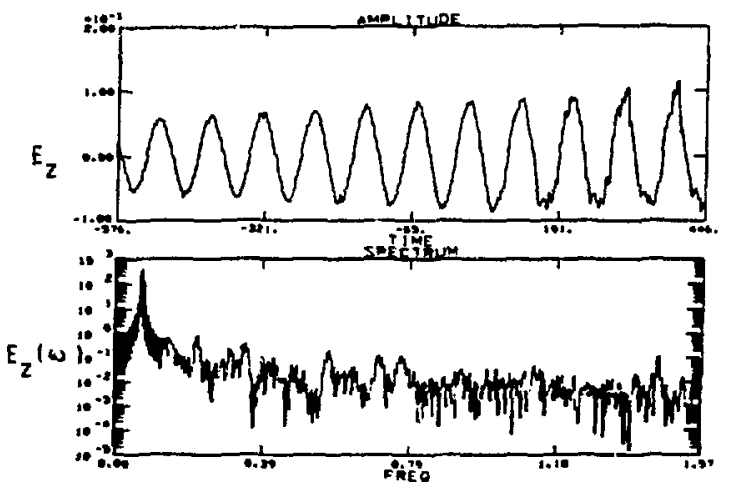

Fig. 1

$E_{2}$ probe trace near center of cavity on-axis. Fields are normalized so that $w=1.0$ corresponds to $51.1 \mathrm{MV} / \mathrm{m}$; frequencies are normalized so that

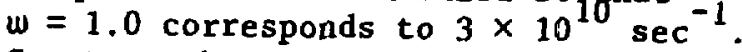
Spectrum shows that signal at $w=0.07$ (350 MHz) is two orders of magnitude above noise or other modes.

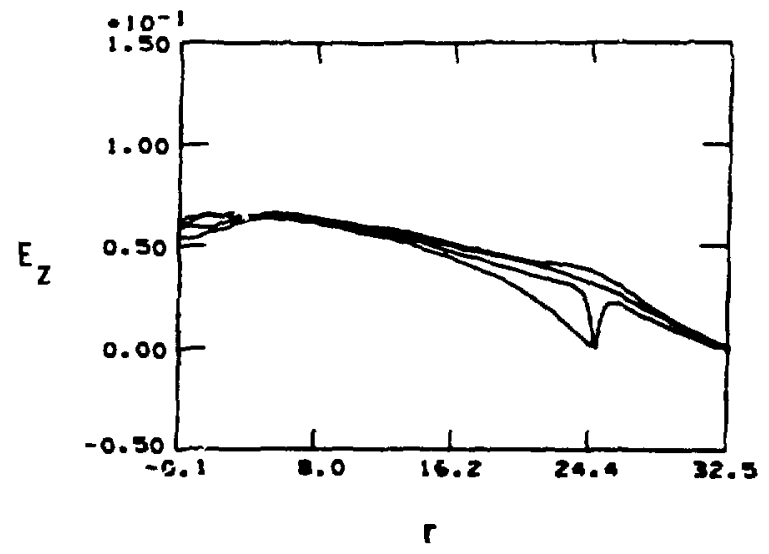

Fig. 2

Radial profile of $E$ cavity fields at four different $z$-positions (superimposed) at a givell instant. Perturbation due to the antenna at $R=25 \mathrm{~cm}$ is clearly observable. 

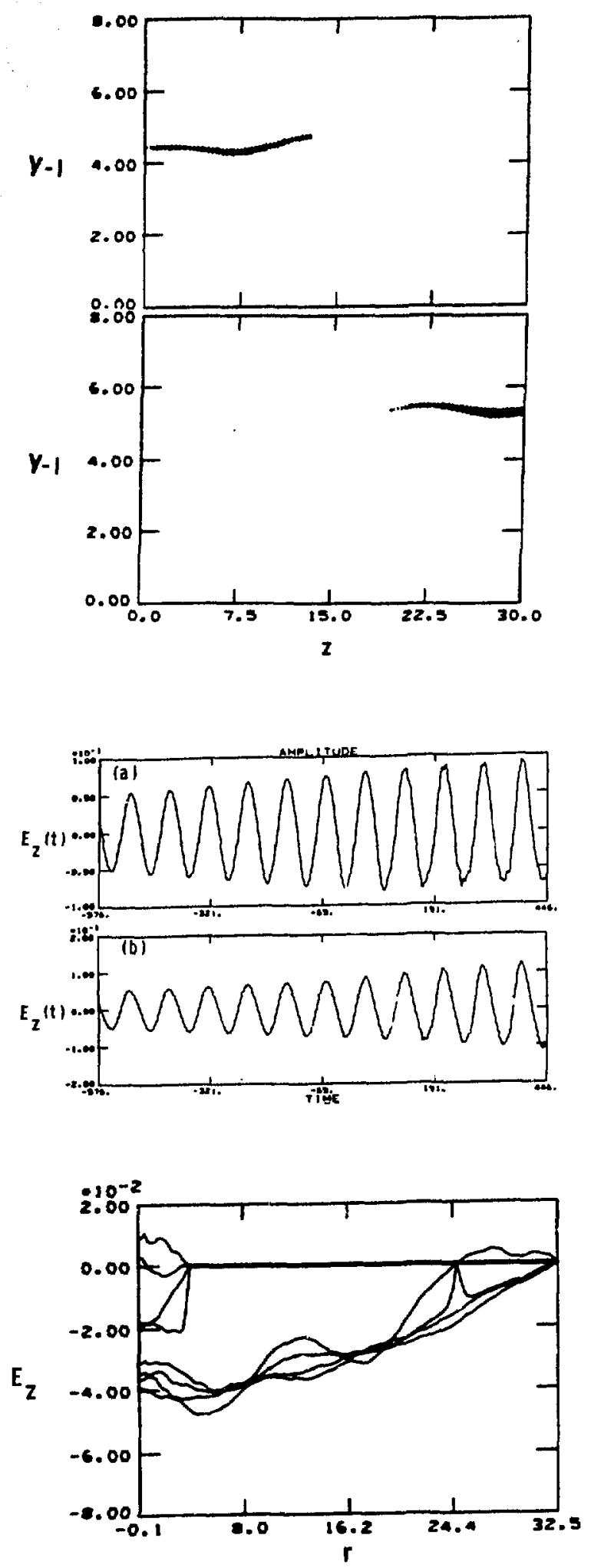

Fig. 3

Energy space of a given micropulse of electrons before and after transit through cavity. Energy gain of $0.5 \mathrm{MeV}$ is accompanied by spacecharge depression and non-uniform acceleration.
Fig. 4

Comparison of $\mathrm{E}$ probe traces near center of cavity at $R=8.7 \mathrm{~cm}$ :

(a) pulses injected in phase with accelerating gradient, (b) pulses injected out of phase with accelerating gradient. Note that field difference is such that the autoaccelerator mode (b) has almost $1.5 \mathrm{MV} / \mathrm{m}$ larger fields by $w_{p} t=445$ (0.5 micropulses).
Fig. 5

Radial profile similar to that in Fig. 2, except profiles of fringing fields are also included. Superposition of higher order cavity fields on the fundamental is evident. 